Scott R. Plotkin, MD,

$\mathrm{PhD}$

Stephanie D. Davis, MD

Kent A. Robertson, MD

Srivandana Akshintala,

MBBS, MPH

Julian Allen, MD

Michael J. Fisher, MD

Jaishri O. Blakeley, MD

Brigitte C. Widemann, MD

Rosalie E. Ferner, MD

Carole L. Marcus, $\mathrm{MBBCh}$

For the REiNS

International

Collaboration

Correspondence to

Dr. Plotkin:

splotkin@partners.org

Supplemental data at Neurology.org

\title{
Sleep and pulmonary outcomes for clinical trials of airway plexiform neurofibromas in NF1
}

\section{ABSTRACT}

Objective: Plexiform neurofibromas (PNs) are complex, benign nerve sheath tumors that occur in approximately $25 \%-50 \%$ of individuals with neurofibromatosis type 1 (NF1). PNs that cause airway compromise or pulmonary dysfunction are uncommon but clinically important. Because improvement in sleep quality or airway function represents direct clinical benefit, measures of sleep and pulmonary function may be more meaningful than tumor size as endpoints in therapeutic clinical trials targeting airway PN.

Methods: The Response Evaluation in Neurofibromatosis and Schwannomatosis functional outcomes group reviewed currently available endpoints for sleep and pulmonary outcomes and developed consensus recommendations for response evaluation in NF clinical trials.

Results: For patients with airway PNs, polysomnography, impulse oscillometry, and spirometry should be performed to identify abnormal function that will be targeted by the agent under clinical investigation. The functional group endorsed the use of the apnea hypopnea index (AHI) as the primary sleep endpoint, and pulmonary resistance at $10 \mathrm{~Hz}\left(\mathrm{R}_{10}\right)$ or forced expiratory volume in 1 or 0.75 seconds $\left(\mathrm{FEV}_{1}\right.$ or $\mathrm{FEV}_{0.75}$ ) as primary pulmonary endpoints. The group defined minimum changes in $A H I, R_{10}$, and $F E V_{1}$ or $F E V_{0.75}$ for response criteria. Secondary sleep outcomes include desaturation and hypercapnia during sleep and arousal index. Secondary pulmonary outcomes include pulmonary resistance and reactance measurements at 5, 10, and $20 \mathrm{~Hz}$; forced vital capacity; peak expiratory flow; and forced expiratory flows.

Conclusions: These recommended sleep and pulmonary evaluations are intended to provide researchers with a standardized set of clinically meaningful endpoints for response evaluation in trials of NF1-related airway PNs. Neurology ${ }^{\circledR}$ 2016;87 (Suppl 1):S13-S20

\section{GLOSSARY}

AASM = American Academy of Sleep Medicine; $\mathbf{A H I}=$ apnea hypopnea index; $\mathbf{C P A P}=$ continuous positive airway pressure; FEV $_{0.75}=$ forced expiratory volume in 0.75 seconds; FEV $_{\mathbf{1}}=$ forced expiratory volume in 1 second; $\mathbf{F V C}=$ forced vital capacity; NF1 = neurofibromatosis type 1; OSA = obstructive sleep apnea; PEF = peak expiratory flow; $\mathbf{P N}=$ plexiform neurofibroma; PSG = polysomnography; REiNS = Response Evaluation in Neurofibromatosis and Schwannomatosis.

Plexiform neurofibromas (PNs) are complex, benign nerve sheath tumors that occur in approximately $25 \%-50 \%$ of individuals with neurofibromatosis type 1 (NF1). The frequency and type of $\mathrm{PN}$-associated morbidities are influenced by tumor location and volume (figure). ${ }^{1-7} \mathrm{~A}$ total of $15 \%-38 \%$ of all PNs occur in the head/neck region and 6\%-25\% occur in the thorax/mediastinum. Airway PNs are a subset of PNs that arise in close proximity to the airway. Symptomatic $\mathrm{PNs}$ that cause airway compromise or pulmonary dysfunction are uncommon (reported in $5 \%-7 \%$ of children with PNs) but clinically important. If left untreated, these lesions can be fatal. Surgical resection is often not feasible, and tracheostomy is required in some patients. ${ }^{3,5}$

Obstructive sleep apnea (OSA) is caused by repeated collapse of the airway during sleep resulting in partial obstruction (hypopnea) or total obstruction (apnea). In the general pediatric

\footnotetext{
From the Neurology Department and Cancer Center (S.R.P.), Massachusetts General Hospital, Boston; Section of Pediatric Pulmonology, Allergy and Sleep Medicine (S.D.D.), and Stem Cell Transplantation Program (K.A.R.), Riley Children's Hospital, Indiana University School of Medicine, Indianapolis; Pediatric Oncology Branch (S.A., B.C.W.), National Cancer Institute, Bethesda, MD; Division of Pulmonary Medicine (J.A.), Division of Oncology (M.J.F.), and Sleep Center (C.L.M.), Children's Hospital of Philadelphia; Department of Pediatrics (M.J.F.) and Sleep Center (C.L.M.), The Perelman School of Medicine at the University of Pennsylvania (J.A.), Philadelphia; Department of Neurology (J.O.B.), John Hopkins Medical Institute, Baltimore, MD; and Department of Neurology (R.E.F.), Guy's and St. Thomas' NHS Foundation Trust and Institute of Psychiatry, King's College London, UK.

REiNS International Collaboration members are listed on the Neurology ${ }^{\circledR}$ Web site at Neurology.org.

Go to Neurology.org for full disclosures. Funding information and disclosures deemed relevant by the authors, if any, are provided at the end of the article.
} 
plus hypopneas/h of sleep. The American Academy of Sleep Medicine (AASM) provides
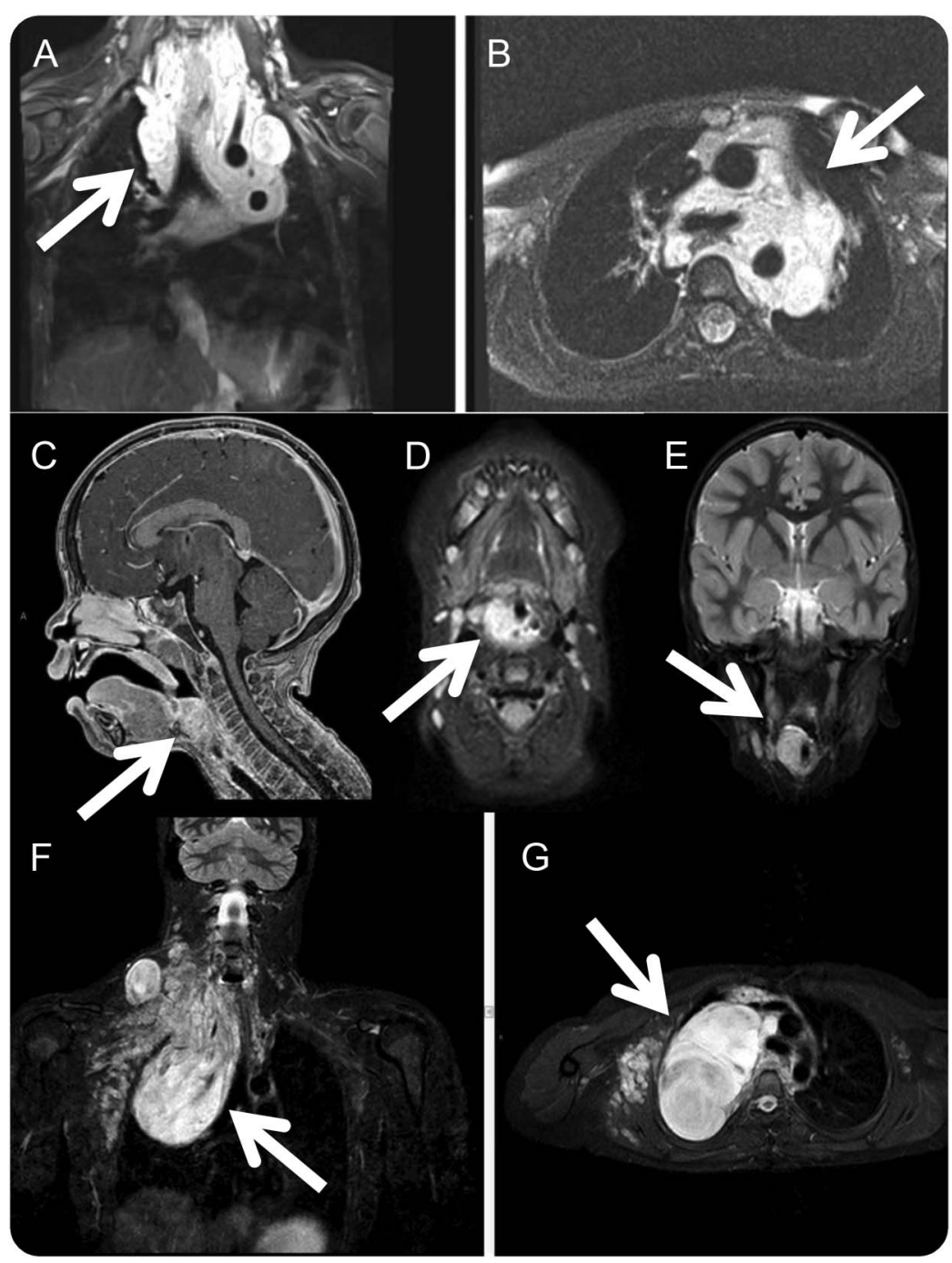

E
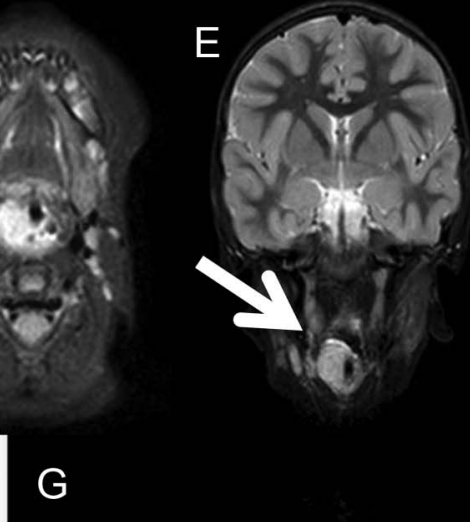

G

Coronal (A) and axial (B) MRI of a large neck/mediastinal PN in a child. Functional improvement would be a more clinically meaningful endpoint compared to imaging response (defined as $a \geq 20 \%$ decrease in the entire $P N$ volume) as even a small volume change could result in clinical benefit. Sagittal (C), axial (D), and coronal (E) MRI of an extrathoracic pharyngeal PN in a child who presented with snoring. Oscillometry should serve best to monitor changes in pulmonary function. Coronal (F) and axial (G) MRI of a predominantly intrathoracic chest PN in a child. Spirometry should serve best to monitor changes in pulmonary function.

population, risk factors include adenotonsillar hypertrophy, obesity, airway encroachment, abnormal respiratory control, and dysfunctional upper airway muscles. Symptoms include snoring, pauses in breathing, and excessive sleepiness. However, there is only a modest correlation between OSA symptoms and objectively measured polysomnographic parameters of OSA severity. ${ }^{8,9}$ Overnight polysomnography (PSG) is the study of choice for patients with suspected OSA. ${ }^{10,11}$ The most widely used measure of PSG is the apnea hypopnea index $(\mathrm{AHI})$, the number of apneas
2 definitions of hypopneas for adults: the definition requiring 3\% desaturation or arousal should be used; this definition is consistent with the pediatric definition. ${ }^{12}$ In children, a normal AHI is $<1.5$. In adults, a normal $\mathrm{AHI}$ is $<5$; the degree of OSA can be classified as mild (AHI 5-14), moderate (AHI 15-30), or severe (AHI >30). Treatment of OSA is directed at maintaining patency of the upper airway. Adenotonsillectomy is the standard treatment for childhood OSA, ${ }^{11}$ and continuous positive airway pressure (CPAP) is the standard treatment if surgery is not an option.

Pulmonary function tests are used to evaluate patients with airway and lung disease. Impulse oscillometry measures airway mechanics by applying pressure oscillations that propagate throughout the airway as the patient tidal breathes in a stable pattern. It is used to measure pulmonary resistance (pressure required to propagate flow through the respiratory system) and reactance (pressure required to overcome elastic and inertive properties of the respiratory system). ${ }^{13}$ Spirometry measures the amount (volume) or speed (flow) of air that can be inhaled and exhaled. Forced vital capacity (FVC) is the maximal volume of air exhaled from full inspiration.

Previously, the Response Evaluation in Neurofibromatosis and Schwannomatosis (REiNS) group published recommendations for hearing and facial function outcomes. ${ }^{14}$ In this report, the group proposes consensus recommendations for sleep and pulmonary outcomes in clinical trials that target airway PNs. In recent years, shrinkage of PNs has been observed after treatment with PEG-intron, imatinib, and selumetinib. ${ }^{17-17}$ Because clinical benefit may occur with minimal volume changes, sleep and pulmonary outcomes may be more sensitive than radiographic changes at detecting a meaningful response to treatment. Our objectives were to define reproducible and clinically meaningful measures of sleep and pulmonary function, which can be used as primary endpoints in clinical trials targeting airway PNs.

METHODS The REiNS functional outcomes group has approximately 20 active participants including professionals from various disciplines involved in NF care. The group engaged 
experts in sleep and pulmonary medicine (C.L.M., S.D.D., J.A.) to help draft these recommendations, which represent expert consensus based on review of the literature.

RESULTS For patients with airway PNs, overnight PSG, impulse oscillometry, and spirometry should be performed during screening to identify abnormal function. Based on performance on these studies, investigators should select a primary measure that will serve as the functional endpoint of interest for each patient. Once a measure has been selected as the primary functional endpoint, it should be tested consistently during a clinical trial with reference to baseline.

Recommended PSG outcomes. For clinical trial outcomes, the group considered the following measures as primary or secondary endpoints: AHI, oxygen saturation, end tidal $\mathrm{CO}_{2}$, and arousal index (table 1). The group recommended use of $\mathrm{AHI}$ as primary outcome because it (1) is a clinically meaningful measure of upper airway function; (2) is widely available at most centers; (3) is the most commonly used endpoint in both pediatric and adult sleep studies, including the effects of adenotonsillectomy, upper airway stimulation, and bariatric surgery for OSA ${ }^{18-20}$; and (4) has been validated in relation to neurocognitive and cardiovascular outcomes of OSA. ${ }^{21,22}$ To date, no studies using AHI for patients with NF1 with airway PNs have been published.

Feasibility. PSG is widely available in most developed countries and a full-night study should be performed in a sleep laboratory. ${ }^{23,24}$ The detailed parameters, settings, filters, technical specifications, and event scoring should be done in accordance with the guidelines in the AASM Manual for the Scoring of Sleep and Associated Events. ${ }^{12}$ Note that the criteria for scoring of obstructive apnea in children differ from those in adults.

Patient characteristics. PSG has been validated in adults and in children, with standardized norms available for children. Physical examination should be performed to document obesity and signs of upper airway narrowing such as tonsillar hypertrophy, retrognathia, macroglossia, enlarged uvula, abnormal hard palate, and nasal abnormalities. Potential participants should be evaluated by a qualified practitioner prior to enrollment to determine whether adenotonsillectomy may result in sufficient improvement in airway function. Patients who meet the criteria for baseline AHI noted below may be considered eligible for trials; patients with an AHI $<5$ would be ineligible based on the criteria defined for improvement. Patients with tracheostomy are not eligible for trials with primary sleep outcomes but may be appropriate for trials with primary imaging outcomes.

Currently, the group does not endorse the use of home sleep studies in clinical trials. This recommendation is based on the paucity of validation studies for children, the limited number of EEG channels in home studies, and the lack of reliable $\mathrm{CO}_{2}$ monitoring (which is important for identifying individuals with hypoventilation). This recommendation should be reconsidered as additional data on home sleep studies in young children become available.

Sleep efficiency (total sleep time as a percentage of total recording time), the amount of REM sleep as a percentage of total sleep time, and body position should be measured to assess the adequacy of PSG. If a patient sleeps very poorly in the laboratory setting and has decreased total sleep time or REM sleep time, the AHI may appear artificially low. Body position

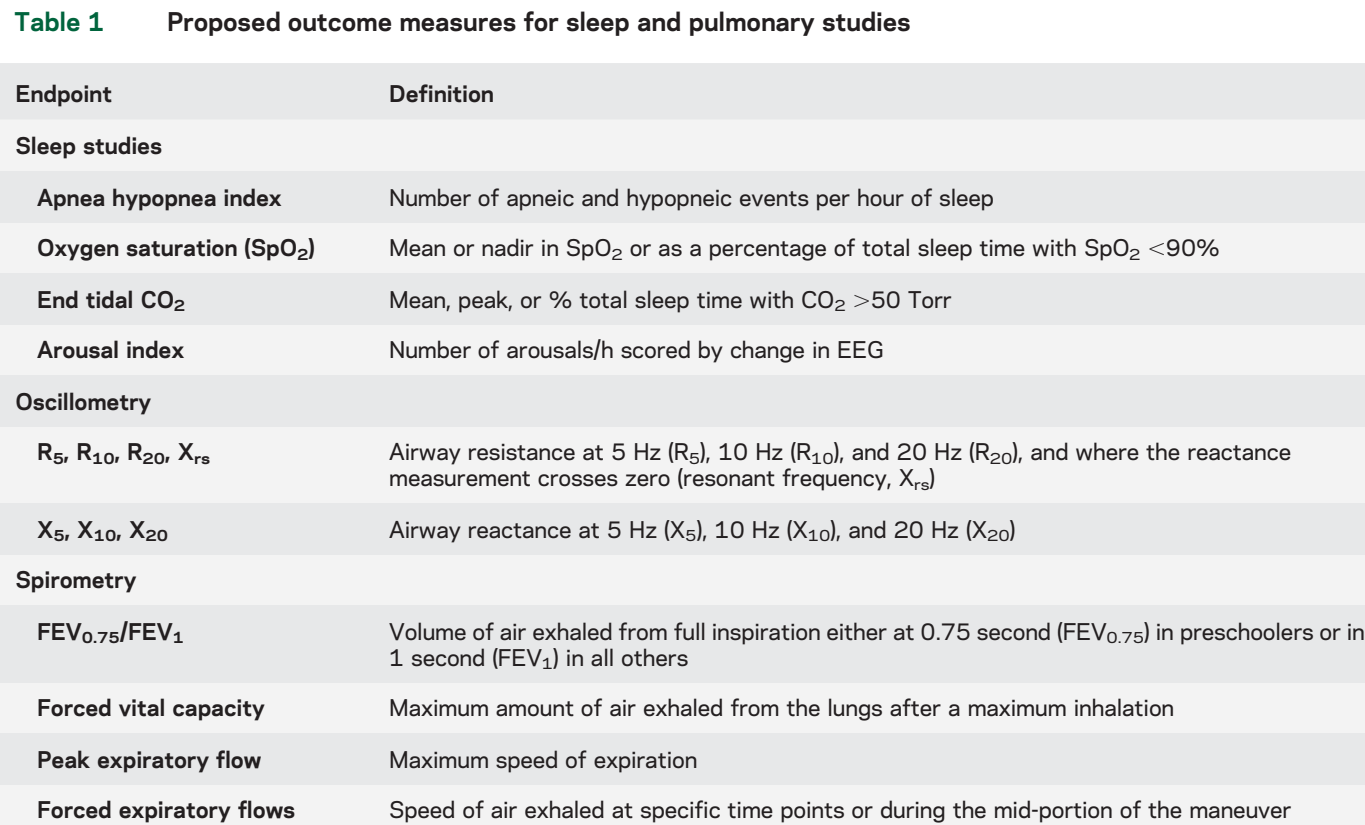

Apnea hypopnea index

Oxygen saturation $\left(\mathrm{SpO}_{2}\right)$

End tidal $\mathrm{CO}_{2}$

Arousal index

Oscillometry

$\mathbf{R}_{5}, \mathbf{R}_{10}, \mathbf{R}_{\mathbf{2 0}}, \mathbf{X}_{\mathrm{rs}}$

$X_{5}, X_{10}, X_{20}$

Spirometry

$\mathrm{FEV}_{0.75} / \mathrm{FEV}_{1}$

Forced vital capacity

Peak expiratory flow

Forced expiratory flows

Number of apneic and hypopneic events per hour of sleep

Mean or nadir in $\mathrm{SpO}_{2}$ or as a percentage of total sleep time with $\mathrm{SpO}_{2}<90 \%$

Mean, peak, or \% total sleep time with $\mathrm{CO}_{2}>50$ Torr

Number of arousals/h scored by change in EEG

Airway resistance at $5 \mathrm{~Hz}\left(\mathrm{R}_{5}\right), 10 \mathrm{~Hz}\left(\mathrm{R}_{10}\right)$, and $20 \mathrm{~Hz}\left(\mathrm{R}_{20}\right)$, and where the reactance measurement crosses zero (resonant frequency, $\mathrm{X}_{\mathrm{rs}}$ )

Airway reactance at $5 \mathrm{~Hz}\left(\mathrm{X}_{5}\right), 10 \mathrm{~Hz}\left(\mathrm{X}_{10}\right)$, and $20 \mathrm{~Hz}\left(\mathrm{X}_{20}\right)$

Volume of air exhaled from full inspiration either at 0.75 second $\left(F E V_{0.75}\right)$ in preschoolers or in 1 second $\left(F E V_{1}\right)$ in all others

Maximum amount of air exhaled from the lungs after a maximum inhalation

Maximum speed of expiration

Speed of air exhaled at specific time points or during the mid-portion of the maneuver 


\begin{tabular}{|c|c|c|c|c|}
\hline Table 2 & $\begin{array}{l}\text { Propose } \\
\text { studies }\end{array}$ & d respons & criteria for & sleep \\
\hline $\begin{array}{l}\text { Baseline } \\
\text { AHI }\end{array}$ & $\begin{array}{l}\text { Events per } \\
8 \text { hours of } \\
\text { sleep }\end{array}$ & $\begin{array}{l}\text { Response } \\
\text { (AHI) }\end{array}$ & $\begin{array}{l}\text { Progression } \\
\text { (AHI) }\end{array}$ & $\begin{array}{l}\text { Stable } \\
\text { (AHI) }\end{array}$ \\
\hline$\leq 5$ & $\begin{array}{l}\text { Not eligible } \\
\text { text) }\end{array}$ & for trials due & to ceiling effec & ct (see \\
\hline 6 & 48 & $\leq 1$ & $\geq 11$ & $2-10$ \\
\hline 7 & 56 & $\leq 2$ & $\geq 12$ & 3-11 \\
\hline 8 & 64 & $\leq 3$ & $\geq 13$ & $4-12$ \\
\hline 9 & 72 & $\leq 4$ & $\geq 14$ & $5-13$ \\
\hline 10 & 80 & $\leq 5$ & $\geq 15$ & $6-14$ \\
\hline 11 & 88 & $\leq 6$ & $\geq 16$ & $7-15$ \\
\hline 12 & 96 & $\leq 7$ & $\geq 17$ & $8-16$ \\
\hline 13 & 104 & $\leq 8$ & $\geq 18$ & $9-17$ \\
\hline 14 & 112 & $\leq 9$ & $\geq 19$ & $10-18$ \\
\hline 15 & 120 & $\leq 10$ & $\geq 20$ & $11-19$ \\
\hline 16 & 128 & $\leq 11$ & $\geq 21$ & $12-20$ \\
\hline 17 & 136 & $\leq 12$ & $\geq 22$ & $13-21$ \\
\hline 18 & 144 & $\leq 13$ & $\geq 23$ & $14-22$ \\
\hline 19 & 152 & $\leq 14$ & $\geq 24$ & $15-23$ \\
\hline 20 & 160 & $\leq 15$ & $\geq 25$ & $16-24$ \\
\hline 21 & 168 & $\leq 15$ & $\geq 27$ & $16-26$ \\
\hline 22 & 176 & $\leq 15$ & $\geq 29$ & $16-28$ \\
\hline 23 & 184 & $\leq 15$ & $\geq 31$ & $16-30$ \\
\hline 24 & 192 & $\leq 15$ & $\geq 33$ & $16-32$ \\
\hline 25 & 200 & $\leq 15$ & $\geq 35$ & $16-34$ \\
\hline 26 & 208 & $\leq 16$ & $\geq 36$ & $17-35$ \\
\hline 27 & 216 & $\leq 17$ & $\geq 37$ & $18-36$ \\
\hline 28 & 224 & $\leq 18$ & $\geq 38$ & $19-37$ \\
\hline 29 & 232 & $\leq 19$ & $\geq 39$ & $20-38$ \\
\hline 30 & 240 & $\leq 20$ & $\geq 40$ & $21-39$ \\
\hline 31 & 248 & $\leq 21$ & $\geq 41$ & $22-40$ \\
\hline 32 & 256 & $\leq 22$ & $\geq 42$ & $23-41$ \\
\hline 33 & 264 & $\leq 23$ & $\geq 43$ & $24-42$ \\
\hline 34 & 272 & $\leq 24$ & $\geq 44$ & $25-43$ \\
\hline 35 & 280 & $\leq 25$ & $\geq 45$ & $26-44$ \\
\hline 36 & 288 & $\leq 26$ & $\geq 46$ & $27-45$ \\
\hline 37 & 296 & $\leq 27$ & $\geq 47$ & $28-46$ \\
\hline 38 & 304 & $\leq 28$ & $\geq 48$ & $29-47$ \\
\hline 39 & 312 & $\leq 29$ & $\geq 49$ & $30-48$ \\
\hline 40 & 320 & $\leq 30$ & $\geq 50$ & $31-49$ \\
\hline 41 & 328 & $\leq 31$ & $\geq 51$ & $32-50$ \\
\hline 42 & 336 & $\leq 32$ & $\geq 52$ & 33-51 \\
\hline 43 & 344 & $\leq 33$ & $\geq 53$ & $34-52$ \\
\hline 44 & 352 & $\leq 34$ & $\geq 54$ & $35-53$ \\
\hline 45 & 360 & $\leq 35$ & $\geq 55$ & $36-54$ \\
\hline 46 & 368 & $\leq 36$ & $\geq 56$ & $37-55$ \\
\hline
\end{tabular}

\begin{tabular}{|lllll|}
\hline Table 2 & \multicolumn{2}{c|}{ Continued } \\
$\begin{array}{lllll}\text { Baseline } \\
\text { AHI }\end{array}$ & $\begin{array}{l}\text { Events per } \\
8 \text { hours of } \\
\text { sleep }\end{array}$ & $\begin{array}{l}\text { Response } \\
\text { (AHI) }\end{array}$ & $\begin{array}{l}\text { Progression } \\
\text { (AHI) }\end{array}$ & $\begin{array}{l}\text { Stable } \\
\text { (AHI) }\end{array}$ \\
47 & 376 & $\leq 37$ & $\geq 57$ & $38-56$ \\
48 & 384 & $\leq 38$ & $\geq 58$ & $39-57$ \\
49 & 392 & $\leq 39$ & $\geq 59$ & $40-58$ \\
250 & \multicolumn{4}{l}{ Not eligible for trials due to floor effect (see text) }
\end{tabular}

Note that values for individual patients should be calculated according to the baseline apnea hypopnea index (AHI) at study initiation.

may affect the severity of OSA. While this is generally less of an issue in children than adults, ${ }^{25}$ it may be important in patients with airway tumors. Serial studies with large differences in the percentage of time in different body positions should be interpreted with caution. Protocols should provide explicit guidance for assessing adequacy of sleep studies in clinical trials. In general, a PSG is considered adequate if there are at least 6 hours of total sleep time (as measured by EEG, not total recording time) and at least an hour of REM sleep (as most sleep-disordered breathing occurs during REM sleep).

Response criteria. AHI response criteria are defined in reference to the baseline AHI at study initiation. Studies of night-to-night variation in AHI suggest that individuals with higher AHI at baseline demonstrate more variability than individuals with lower AHI. ${ }^{26,27}$ Thus, the group recommended a larger absolute change in AHI for patients with higher baseline AHI in order to minimize the rate of falsepositive or false-negative responses. ${ }^{26,27}$ Additional studies on night-to-night variation in AHI for patients with NF1 with airway $\mathrm{PN}$ are needed to refine these response criteria. Until these studies are complete, the functional group recommends using criteria based on expert opinion (table 2).

Functional improvement is defined as an absolute decrease in AHI by $\geq 5$ events/h (for patients with baseline $\mathrm{AHI} \leq 20$ ) or an absolute decrease in AHI by $\geq 10$ events/h (for patients with baseline AHI $\geq 25$ ). Functional decline is defined as an absolute increase in AHI by $\geq 5$ events/h (for patients with baseline AHI $\leq 20$ ) or an absolute increase in AHI by $\geq 10$ events/h (for patients with baseline AHI $\geq 25$ ). See table 2 for recommendations when the baseline AHI is $21-24$. Stable function is defined as all other responses. Table 2 outlines the amount of change in AHI that represents response or progression based on baseline AHI.

Special notes on AHI. AHI is a bounded variable: it is an average number of events during an overnight sleep study (typically 8 hours or more) and the 
variable ranges from 0 (no events) to $>100$ events $/ \mathrm{h}$. The existence of upper and lower boundaries introduces a ceiling and floor effect for patients with values near the top and bottom of the range, respectively. As table 2 shows, patients with an $\mathrm{AHI} \leq 5$ /hour are not eligible for response since these patients cannot improve enough to meet criteria for functional improvement. The functional group recommends that patients with an $\mathrm{AHI} \geq 50$ /hour should not be enrolled in clinical trials at this time given the availability of alternative treatments for very severe OSA.

Patients treated with CPAP should undergo PSG off CPAP if they are clinically stable to do so, as judged by their medical team. PSG on the first night off CPAP may show milder OSA than on subsequent nights. ${ }^{28-30}$ Because of this transient improvement, CPAP should be discontinued for 48 hours prior to PSG. During this time, patients should be monitored for clinical exacerbations. The research polysomnogram should be discontinued for any event that the research team considers unsafe, such as pathologic cardiac arrhythmia during sleep other than sinus arrhythmia.

To ensure the safety of patients during subsequent sleep studies, the group proposes that patients who meet the following parameters during a study should be declared as functional decline and CPAP should be reinstituted to meet safety needs: any condition for which the research team considers the patient medically unstable, such as treatment-emergent pathologic cardiac arrhythmia during sleep other than sinus arrhythmia not explained by electrolyte abnormalities.

Secondary outcomes for sleep studies. Alternative measures of sleep function are available from a standard PSG. These measures include the change in (1) oxygen saturation $\left(\mathrm{SpO}_{2}\right)$ measured as mean or nadir $\mathrm{SpO}_{2}$, or as percentage of total sleep time with $\mathrm{SpO}_{2}<90 \%$; (2) end tidal $\mathrm{CO}_{2}$ measured as mean or peak $\mathrm{CO}_{2}$, or as percentage of total sleep time with $\mathrm{CO}_{2}>50$ Torr; and (3) arousal index, defined as the number of arousals per hour scored by EEG. While the group committee does not endorse changes in these variables as a primary outcome measure, we do recommend that all of these variables, and possibly other measures such as neurocognitive, imaging, patient-reported, or quality of life measures, be recorded as secondary outcomes to provide additional data about the effectiveness of the intervention.

Recommended outcome for pulmonary function. For patients in whom pulmonary function is the primary endpoint of interest, investigators should select either oscillometry or spirometry as the primary measure based on physiologic findings. In general, the functional group endorsed the use of oscillometry for pulmonary studies of extrathoracic/upper airway lesions and spirometry for pulmonary studies of intrathoracic/peripheral airway lesions. Oscillometry has been used as a primary or secondary endpoint in studies on adenoidectomy for exercise-induced bronchoconstriction, in a prospective trial of a multifaceted intervention to decrease asthma onset or severity, and in a study evaluating pulmonary exacerbation response in cystic fibrosis. ${ }^{31-33}$ The following measures were considered as potential endpoints for impulse oscillometry: $R_{5}, R_{10}, R_{20}$, $\mathrm{X}_{5}, \mathrm{X}_{10}, \mathrm{X}_{20}$, and changes in resistance at the resonant frequency (table 1). The group recommended resistance at $10 \mathrm{~Hz}\left(\mathrm{R}_{10}\right)$ as the primary outcome measure for oscillometry. A pulmonary resistance measure $(\mathrm{R})$ was preferred over a pulmonary reactance measure $(\mathrm{X})$ since airway $\mathrm{PNs}$ are more likely to impact the resistance properties than the elastic and inertive properties of airways. Further, $\mathrm{R}_{10}$ was selected among the resistance measures since $R_{10}$ is a better measure of central airways compared with $\mathrm{R}_{5}$ and has been used in more trials as an outcome measure compared to $R_{20}$.

For spirometry, forced expiratory volume in 0.75 seconds $\left(\mathrm{FEV}_{0.75}\right)$ (in preschool children), forced expiratory volume in 1 second $\left(\mathrm{FEV}_{1}\right)$ (in all others), FVC, and peak expiratory flow (PEF) were considered as potential endpoints (table 1 ). $\mathrm{FEV}_{1}$ has been used as a primary endpoint or secondary endpoint in a number of clinical trials for asthma ${ }^{34}$ and cystic fibrosis. ${ }^{35}$ The group recommended $\mathrm{FEV}_{0.75}$ for preschool children and $\mathrm{FEV}_{1}$ for all others as primary outcome measure for spirometry. FEV 0.75 and $\mathrm{FEV}_{1}$ were preferred since these measures reflect airway obstruction, have low variability on repeat testing, and are widely accepted as primary outcomes for studies of children with airway disease.

Feasibility. Impulse oscillometry is available at many large medical centers but is not standard at all medical centers. Children should be instructed to breathe normally during the test and to avoid crossing the legs as this position can contract the abdominal wall and lead to diminished resting end-expiratory pulmonary volumes. Further, the cheeks must be firmly supported by hands to compensate for cheek compliance. In general, a 30-second interval of testing is performed, and an average of 3-5 measurements is obtained for analysis. Spirometry is widely available for clinical trials. Studies should be attended by trained personnel in order to monitor for technical adequacy and patient compliance. For both spirometry and impulse oscillometry, equipment quality control and calibration are essential for accurate and valid testing. The detailed parameters and technical specifications should be performed as described in the Standardization of Spirometry published by the American Thoracic Society. ${ }^{36}$ Additional guidelines for young children are also available. ${ }^{37}$

Patient characteristics. Impulse oscillometry is commonly employed in children since it requires only 
passive cooperation of the patient (in contrast to spirometry, which requires active participation of the patient). Standardized normative values for adults and children down to 2 years of age have been published. ${ }^{37,38}$ Spirometry is highly dependent on patient cooperation and effort and can only be used in children who are able to follow instructions reliably. In general, spirometry can be performed in children who are 6 years or older; however, cooperative preschoolers are able to produce acceptable results. ${ }^{36,37}$ Normative values for $\mathrm{FEV}_{0.75}$ and $\mathrm{FEV}_{1}$ are available for individuals aged 3-90 years. ${ }^{39-41}$

Response criteria. Response criteria for $\mathrm{R}_{10}$ are defined in reference to the baseline $\mathrm{R}_{10}$ at study initiation. Functional improvement is defined as $\geq 20 \%$ decrease in $\mathrm{R}_{10}$, functional decline is defined as $\geq 20 \%$ increase in $R_{10}$, and stable function is defined as all other responses. A threshold of $20 \%$ was chosen based on data showing the average coefficient of variation for resistance is $\leq 10 \%$ between tests. ${ }^{37}$

Response criteria for $\mathrm{FEV}_{0.75} / \mathrm{FEV}_{1}$ are defined in reference to the baseline $\mathrm{FEV}_{0.75} / \mathrm{FEV}_{1}$ at study initiation. Functional improvement is defined as $\geq 12 \%$ increase in $\mathrm{FEV}_{0.75}$ (preschoolers) or in $\mathrm{FEV}_{1}$ (all others). Functional decline is defined as $\geq 12 \%$ decrease in $\mathrm{FEV}_{0.75}$ (preschoolers) or in $\mathrm{FEV}_{1}$ (all others). Stable function is defined as all other responses. The working group adopted these thresholds based on the recommendation of the American Thoracic Society/European Respiratory Society Task Force. ${ }^{42}$

Special notes on $\boldsymbol{R}_{10}$ and $\boldsymbol{F E V}$. In clinical practice, $\mathrm{R}_{10}$ is an unbounded variable. For clinical trials, the group recommended excluding patients with $R_{10} \leq 120 \%$ predicted for age in order to allow for sufficient improvement on clinical trial to meet response criteria. No recommendation was made for a maximum $\mathrm{R}_{10}$ value for trial participation given lack of published data on this topic. Similarly, FEV is an unbounded variable in clinical practice that can range from $<10 \%$ to $>100 \%$ of predicted for age, sex, and height. For clinical trials, the group recommended including patients with $\mathrm{FEV}_{0.75}$ or $1 \geq 40 \%$ and $\leq 80 \%$ of predicted for age, sex, and height in order to allow for sufficient improvement or decline in a clinical trial to meet response criteria.

Secondary outcomes for pulmonary function. Alternative measures of pulmonary function are available from oscillometry and spirometry. For oscillometry, these measures may include change in resistance measurements at 5 or $20 \mathrm{~Hz}\left(\mathrm{R}_{5}, \mathrm{R}_{20}\right)$, change in reactance measurements at resonant frequency (frequency at which reactance $=0$ ), and change in reactance at 5 , 10, and $20 \mathrm{~Hz}$. For spirometry, these measures may include changes in FVC, PEF, and forced expiratory flows. The committee recommends that all of these variables, and possibly other measures such as imaging, patient-reported, or quality of life measures, be recorded as secondary outcomes to provide additional data about the effectiveness of the intervention under study.

\section{Additional recommendations. Double baseline evaluations.} The group does not recommend the use of double baseline studies for all clinical trials at this time, due to the substantial resources and time required to obtain these evaluations, and the resulting burden to the patient. However, given the unknown variability of the outcome measures for patients with airway $\mathrm{PN}$ and the possibility of regression to the mean, the group recommends that the baseline variability of these measures be determined in a select group of patients to refine the proposed response criteria.

Frequency of reevaluation. No evidence-based guidelines are available to help determine the optimal interval between evaluations. The group recommends reevaluation of primary endpoints (AHI, $\mathrm{R}_{10}$, or $\mathrm{FEV}_{0.75} / \mathrm{FEV}_{1}$ ) every 3-6 months during prolonged treatment, although the group recognizes that the timing of reevaluation may be influenced by the design of each particular study and the agent being tested. In general, evaluation of primary endpoints should be performed at the same interval as imaging studies to facilitate correlation of functional changes with imaging changes in symptomatic PNs.

Confirmatory measurement. Confirmation of functional response is desirable in nonrandomized trials where response is the primary endpoint and in PN trials where the focus is on reducing long-term morbidity rather than on extending survival. In addition, confirmation of response would increase the confidence that a measured response is the result of the intervention and not due to baseline variability. Confirmation of response may not be necessary for randomized trials or for trials where time to progression is the primary endpoint.

Duration of response and stable disease. The duration of functional response is calculated from the date when the primary endpoint first meets criteria for functional response compared with baseline until the first date that the primary endpoint no longer meets criteria for functional response (table 2). Stable function is calculated from the initiation of treatment until the time that primary endpoint meets criteria for functional decline. In order to declare stable function in any study, testing must document stable function for a minimum of time established by the study protocol (generally 6 months). Patients without subsequent evaluation after baseline evaluation should be considered nonevaluable. Importantly, estimates of duration of response and time to progression are influenced by the interval of evaluations. For this reason, protocols should specify the interval between evaluations. 
Proportion free from functional decline. Patients with NF1 with growing PN involving the airway are at risk for worsening of OSA or pulmonary function. In this trial design, freedom from functional decline at specified time points might be an acceptable endpoint to provide evidence of drug activity. Because freedom from decline is liable to bias in uncontrolled phase 2 trials, this variable is best evaluated by a randomized trial.

Reporting best response. For phase 2 studies, response assessment should include all patients in the trial, including nonevaluable patients and those whose treatment deviates from the study protocol. Responses should be categorized as functional improvement, stable function, functional decline, or nonevaluable (e.g., due to death, toxicity, or lack of assessment). For phase 3 studies, functional response may be a primary or secondary endpoint. When functional response is the primary endpoint, the study must include only patients who are capable of functional improvement and all enrolled patients must be analyzed. When functional response is a secondary endpoint, the study may include patients regardless of sleep or pulmonary function. In this design, functional response may also be reported using a predefined subgroup analysis (with only the patients capable of response in the denominator). The study protocol should include the plan to report responses, including any subgroup analyses.

DISCUSSION These recommendations are designed to supply investigators with a shared group of functional endpoints for clinical trials of airway $\mathrm{PN}$ in $\mathrm{pa}-$ tients with NF1. It is hoped that the use of these endpoints will improve the ability to identify active agents and facilitate comparison across studies with different agents. The recommended outcomes have not been prospectively used in NF1 PN trials to date, and the REiNS International Collaboration expects to revisit these recommendations as data from trials of airway PN are published.

\section{AUTHOR CONTRIBUTIONS}

C.L. Marcus: design and conceptualization of the study, collection and interpretation of the data, revising the manuscript. S.D. Davis: conceptualization of the study, collection and interpretation of the data, revising the manuscript. K.A. Robertson: conceptualization of the study, revising the manuscript. S. Akshintala: interpretation of the data, revising the manuscript. J. Allen: interpretation of the data, revising the manuscript. M.J. Fisher: design and conceptualization of the study, interpretation of the data, revising the manuscript. J.O. Blakeley: conceptualization of the study, interpretation of the data, revising the manuscript. B.C. Widemann: conceptualization of the study, interpretation of the data, revising the manuscript. R.E. Ferner: conceptualization of the study, drafting and revising the manuscript. S.R. Plotkin: design and conceptualization of the study, collection and interpretation of the data, drafting and revising the manuscript.

\section{ACKNOWLEDGMENT}

The authors thank Dr. Albert Li for providing data used in the writing of this manuscript and the following collaborators for their participation in the REiNS functional outcomes working group: Elizabeth Schorry,
D. Bradley Welling, David Stevenson, Dusica Babovic-Vuksanovic, D. Gareth Evans, James Tonsgard, and Trent Hummel.

\section{STUDY FUNDING}

No targeted funding reported.

\section{DISCLOSURE}

S. Plotkin: research support from the Children's Tumor Foundation, NIH, Department of Defense Neurofibromatosis Clinical Trials Consortium. S. Davis: research support by NIH, Cystic Fibrosis Foundation; served on advisory board for Vertex Pharmaceuticals and served as a speaker through ABCOMM, Inc. (supported by educational grant from Gilead). K. Robertson: receives research support from Novartis, NIH, Department of Defense, and Leukemia Lymphoma Society Translational Research Program; and serves on medical advisory board for General BioTechnology-Cook Biomedical. S. Akshintala: research support through NCI CCR Intramural Research Program. J. Allen: Editor, Annals of the American Thoracic Society; Question writer, American Board of Pediatrics. M. Fisher: research support from the Children's Tumor Foundation, Department of Defense Neurofibromatosis Clinical Trials Consortium, Thrasher Research Fund, and Dana Foundation; honoraria for speaking at Nemours/Alfred I. duPont Hospital for Children and Rainbow Babies \& Children's Hospital; reimbursement for attending/ speaking/chairing sessions at conferences from Children's Tumor Foundation and The American Austrian Foundation. J. Blakeley: consultant for Abbvie; research support through NCI-CTEP, Children's Tumor Foundation, and GlaxoSmithKline. B. Widemann: research support through NCI CCR Intramural Research Program. R. Ferner: receives funds from the Children's Tumor Foundation, European Neurofibromatosis Association, and Springer publishing group. C. Marcus: research support from Philips Respironics. Go to Neurology.org for full disclosures.

Received November 19, 2015. Accepted in final form May 6, 2016.

\section{REFERENCES}

1. Kim A, Gillespie A, Dombi E, et al. Characteristics of children enrolled in treatment trials for NF1-related plexiform neurofibromas. Neurology 2009;73:1273-1279.

2. Kim A, Dombi E, Tepas K, et al. Phase I trial and pharmacokinetic study of sorafenib in children with neurofibromatosis type I and plexiform neurofibromas. Pediatr Blood Cancer 2013;60:396-401.

3. Needle MN, Cnaan A, Dattilo J, et al. Prognostic signs in the surgical management of plexiform neurofibroma: the Children's Hospital of Philadelphia experience, 19741994. J Pediatr 1997;131:678-682.

4. Nguyen R, Kluwe L, Fuensterer C, Kentsch M, Friedrich RE, Mautner VF. Plexiform neurofibromas in children with neurofibromatosis type 1: frequency and associated clinical deficits. J Pediatr 2011;159:652-655.

5. Prada CE, Rangwala FA, Martin LJ, et al. Pediatric plexiform neurofibromas: impact on morbidity and mortality in neurofibromatosis type 1. J Pediatr 2012;160: 461-467.

6. Dombi E, Solomon J, Gillespie AJ, et al. NF1 plexiform neurofibroma growth rate by volumetric MRI: relationship to age and body weight. Neurology 2007;68:643-647.

7. Korf BR. Plexiform neurofibromas. Am J Med Genet 1999;89:31-37.

8. Rosen CL, Wang R, Taylor HG, et al. Utility of symptoms to predict treatment outcomes in obstructive sleep apnea syndrome. Pediatrics 2015;135:e662-e671.

9. Mitchell RB, Garetz S, Moore RH, et al. The use of clinical parameters to predict obstructive sleep apnea syndrome severity in children: the Childhood Adenotonsillectomy (CHAT) study randomized clinical trial. JAMA Otolaryngol Head Neck Surg 2015;141:130-136. 
10. Kushida CA, Littner MR, Morgenthaler T, et al. Practice parameters for the indications for polysomnography and related procedures: an update for 2005. Sleep 2005;28: 499-521.

11. Marcus CL, Brooks LJ, Draper KA, et al. Diagnosis and management of childhood obstructive sleep apnea syndrome. Pediatrics 2012;130:576-584.

12. Berry RB, Brooks R, Garnaldo CE, et al. The AASM Manual for the Scoring of Sleep and Associated Events: Rules, Terminology, and Technical Specifications. Report No. 2.2. Darien, IL: American Academy of Sleep Medicine; 2015.

13. Komarow HD, Myles IA, Uzzaman A, Metcalfe DD. Impulse oscillometry in the evaluation of diseases of the airways in children. Ann Allergy Asthma Immunol 2011; 106:191-199.

14. Plotkin SR, Ardern-Holmes SL, Barker FG, et al. Hearing and facial function outcomes for neurofibromatosis 2 clinical trials. Neurology 2013;81:S25-S32.

15. Robertson KA, Nalepa G, Yang FC, et al. Imatinib mesylate for plexiform neurofibromas in patients with neurofibromatosis type 1: a phase 2 trial. Lancet Oncol 2012;13: 1218-1224.

16. Jakacki RI, Dombi E, Potter DM, et al. Phase I trial of pegylated interferon-alpha- $2 \mathrm{~b}$ in young patients with plexiform neurofibromas. Neurology 2011;76:265-272.

17. Widemann BC, Marcus LJ, Fisher MJ, et al. Phase I study of the MEK1/2 inhibitor selumetinib (AZD6244) hydrogen sulfate in children and young adults with neurofibromatosis type 1 (NF1) and inoperable plexiform neurofibromas (PNs). J Clin Oncol 2014;32. Abstract.

18. Marcus CL, Moore RH, Rosen CL, et al. A randomized trial of adenotonsillectomy for childhood sleep apnea. N Engl J Med 2013;368:2366-2376.

19. Strollo PJ Jr, Soose RJ, Maurer JT, et al. Upper-airway stimulation for obstructive sleep apnea. N Engl J Med 2014;370:139-149.

20. Dixon JB, Schachter LM, O'Brien PE, et al. Surgical vs conventional therapy for weight loss treatment of obstructive sleep apnea: a randomized controlled trial. JAMA 2012;308:1142-1149.

21. Redline S, Budhiraja R, Kapur V, et al. The scoring of respiratory events in sleep: reliability and validity. J Clin Sleep Med 2007;3:169-200.

22. Wise MS, Nichols CD, Grigg-Damberger MM, et al. Executive summary of respiratory indications for polysomnography in children: an evidence-based review. Sleep 2011;34:389-398AW.

23. Epstein LJ, Kristo D, Strollo PJ Jr, et al. Clinical guideline for the evaluation, management and long-term care of obstructive sleep apnea in adults. J Clin Sleep Med 2009;5:263-276.

24. Aurora RN, Zak RS, Karippot A, et al. Practice parameters for the respiratory indications for polysomnography in children. Sleep 2011;34:379-388.

25. Fernandes do Prado LB, Li X, Thompson R, Marcus CL. Body position and obstructive sleep apnea in children. Sleep 2002;25:66-71.
26. Katz ES, Greene MG, Carson KA, et al. Night-to-night variability of polysomnography in children with suspected obstructive sleep apnea. J Pediatr 2002;140:589-594.

27. Li AM, Wing YK, Cheung A, et al. Is a 2-night polysomnographic study necessary in childhood sleep-related disordered breathing? Chest 2004;126:1467-1472.

28. Sforza E, Lugaresi E. Daytime sleepiness and nasal continuous positive airway pressure therapy in obstructive sleep apnea syndrome patients: effects of chronic treatment and 1-night therapy withdrawal. Sleep 1995;18:195-201.

29. Kribbs NB, Pack AI, Kline LR, et al. Effects of one night without nasal CPAP treatment on sleep and sleepiness in patients with obstructive sleep apnea. Am Rev Respir Dis 1993;147:1162-1168.

30. Boudewyns A, Sforza E, Zamagni M, Krieger J. Respiratory effort during sleep apneas after interruption of longterm CPAP treatment in patients with obstructive sleep apnea. Chest 1996;110:120-127.

31. Mattila PS, Hammaren-Malmi S, Pelkonen AS, et al. Effect of adenoidectomy on respiratory function: a randomised prospective study. Arch Dis Child 2009;94:366-370.

32. Klinnert MD, Liu AH, Pearson MR, et al. Outcome of a randomized multifaceted intervention with low-income families of wheezing infants. Arch Pediatr Adolesc Med 2007;161:783-790.

33. Ren CL, Brucker JL, Rovitelli AK, Bordeaux KA. Changes in lung function measured by spirometry and the forced oscillation technique in cystic fibrosis patients undergoing treatment for respiratory tract exacerbation. Pediatr Pulmonol 2006;41:345-349.

34. Wechsler ME, Kelley JM, Boyd IO, et al. Active albuterol or placebo, sham acupuncture, or no intervention in asthma. N Engl J Med 2011;365:119-126.

35. Ramsey BW, Davies J, McElvaney NG, et al. A CFTR potentiator in patients with cystic fibrosis and the G551D mutation. N Engl J Med 2011;365:1663-1672.

36. Miller MR, Hankinson J, Brusasco V, et al. Standardisation of spirometry. Eur Respir J 2005;26:319-338.

37. Beydon N, Davis SD, Lombardi E, et al. An official American Thoracic Society/European Respiratory Society statement: pulmonary function testing in preschool children. Am J Respir Crit Care Med 2007;175:1304-1345.

38. Dencker M, Malmberg LP, Valind S, et al. Reference values for respiratory system impedance by using impulse oscillometry in children aged 2-11 years. Clin Physiol Funct Imaging 2006;26:247-250.

39. Wang X, Dockery DW, Wypij D, Fay ME, Ferris BG Jr. Pulmonary function between 6 and 18 years of age. Pediatr Pulmonol 1993;15:75-88.

40. Hankinson JL, Odencrantz JR, Fedan KB. Spirometric reference values from a sample of the general U.S. population. Am J Respir Crit Care Med 1999;159:179-187.

41. Quanjer PH, Stanojevic S, Cole TJ, et al. Multi-ethnic reference values for spirometry for the 3-95-yr age range: the global lung function 2012 equations. Eur Respir J 2012;40:1324-1343.

42. Pellegrino R, Viegi G, Brusasco V, et al. Interpretative strategies for lung function tests. Eur Respir J 2005;26:948-968. 


\section{Neurology}

\section{Sleep and pulmonary outcomes for clinical trials of airway plexiform neurofibromas in NF1}

Scott R. Plotkin, Stephanie D. Davis, Kent A. Robertson, et al.

Neurology 2016;87;S13-S20

DOI 10.1212/WNL.0000000000002933

\section{This information is current as of August 15, 2016}

\begin{tabular}{|c|c|}
\hline $\begin{array}{l}\text { Updated Information \& } \\
\text { Services }\end{array}$ & $\begin{array}{l}\text { including high resolution figures, can be found at: } \\
\text { http://n.neurology.org/content/87/7_Supplement_1/S13.full }\end{array}$ \\
\hline Supplementary Material & $\begin{array}{l}\text { Supplementary material can be found at: } \\
\text { http://n.neurology.org/content/suppl/2016/08/15/WNL.0000000000002 } \\
\text { 933.DC1 }\end{array}$ \\
\hline References & $\begin{array}{l}\text { This article cites } 40 \text { articles, } 4 \text { of which you can access for free at: } \\
\text { http://n.neurology.org/content/87/7_Supplement_1/S13.full\#ref-list-1 }\end{array}$ \\
\hline Citations & $\begin{array}{l}\text { This article has been cited by } 2 \text { HighWire-hosted articles: } \\
\text { http://n.neurology.org/content/87/7_Supplement_1/S13.full\#\#otherartic } \\
\text { les }\end{array}$ \\
\hline Subspecialty Collections & $\begin{array}{l}\text { This article, along with others on similar topics, appears in the } \\
\text { following collection(s): } \\
\text { Clinical trials Methodology/study design } \\
\text { http://n.neurology.org/cgi/collection/clinical_trials_methodology_study } \\
\text { design__ } \\
\text { Nerve tumor } \\
\text { http://n.neurology.org/cgi/collection/nerve_tumor } \\
\text { Neurofibromatosis } \\
\text { http://n.neurology.org/cgi/collection/neurofibromatosis }\end{array}$ \\
\hline Permissions \& Licensing & $\begin{array}{l}\text { Information about reproducing this article in parts (figures,tables) or in } \\
\text { its entirety can be found online at: } \\
\text { http://www.neurology.org/about/about_the_journal\#permissions }\end{array}$ \\
\hline Reprints & $\begin{array}{l}\text { Information about ordering reprints can be found online: } \\
\text { http://n.neurology.org/subscribers/advertise }\end{array}$ \\
\hline
\end{tabular}

Neurology ${ }^{\circledR}$ is the official journal of the American Academy of Neurology. Published continuously since 1951, it is now a weekly with 48 issues per year. Copyright @ 2016 American Academy of Neurology. All rights reserved. Print ISSN: 0028-3878. Online ISSN: 1526-632X.

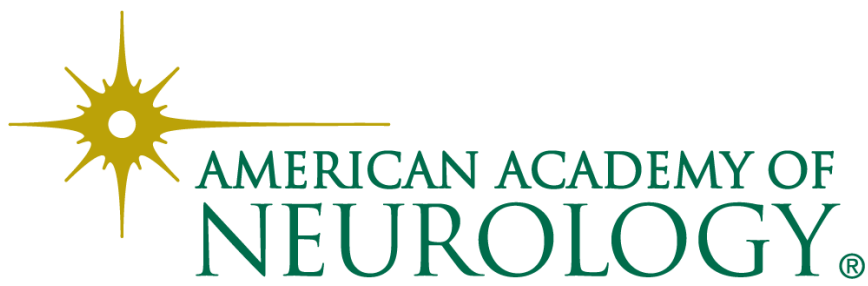

